\title{
COMPUTER AIDED DIAGNOSIS OF GLAUCOMA DETECTION USING DIGITAL FUNDUS IMAGE
}

\author{
Ganeshbabu T R, \\ Department of Electronics and Communication Engineering, \\ Shri Andal Alagar College of Engineering, \\ Mamandur, India \\ ganeshbabutr@gmail.com
}

\begin{abstract}
A robust and cost-effective mass screening may help to detect glaucoma at the earliest which is a major cause of blindness. In this paper, a Computer Aided Diagnosis (CAD) approach for glaucoma detection using retinal fundus images based on clustering techniques is presented. The abnormalities in retinal fundus image are diagnosed using the physiological characteristics of Optic Cup (OC) and Optic Disc (OD). Based on the size of OC and OD, Cup to Disc Ratio (CDR) is computed for the diagnosis. Due to glaucoma, the size of OC increases which increases the CDR as well. In this study, the OD segmentation is achieved by $\mathrm{K}$ - Means clustering (KMC) and Hill Climbing Algorithm (HCA) for the selection of $\mathrm{K}$ value. Similarly, OC is extracted by exploiting fuzzy C-mean clustering. After segmentation of OC and $\mathrm{OD}, \mathrm{CDR}$ is computed to diagnose glaucoma. The system is applied to a total of 45 images, and the results indicate the ability of the system for automated mass screening to diagnose glaucoma at the earliest.
\end{abstract}

Keywords: Glaucoma, Fundus, Optic Cup, Optic Disc, CDR.

\section{INTRODUCTION}

The major cause of blindness is glaucoma which is a series eye disease in the world. It persists for a long time (chronic disease) and around 90\% cases are chronic in nature. As it does not provide any symptoms, it is necessary to check more than two times in a year. The main cause of glaucoma is the pressure inside the eye which is called as intraocular pressure [2]. This pressure damages optic nerve. There are many parameters available to diagnose glaucoma [3].

Chan-Vese model and shape restraint based segmentation of papilla is discussed in [4] using fundus images. The papilla is detected with high accuracy so that the power of discrimination in the extracted shape-based features is very good. Also, it is very robust to noise.

Glaucoma detection based on vessel diameter variation is illustrated in [5]. At first, the registration is done with chamfer matching algorithm, and then differential calculus is employed to detect the vessel centerlines. These profiles are modelled by a Gaussian function to detect vessel diameters.

Morphological based OD localization is discussed in [6] for glaucoma diagnosis. The main circular feature is detected with Hough transform along with gradient image in the horizontal direction. Initially, the brightest areas are isolated by morphological operations.

Level set based CDR computation using fundus images is described in [7]. It uses variational level set and threshold level set for OD and OC segmentation respectively. Finally, the contours are smoothed by elliptical fitting. CDR for glaucoma diagnosis is described in [8] using retinal fundus images based on variational level set and threshold approaches. 
In this study, CDR is used for the diagnosis using retinal fundus images. Fundus images show the various characteristics of the retina such as macula, OC and OD. A sample fundus image is shown in Figure 1.

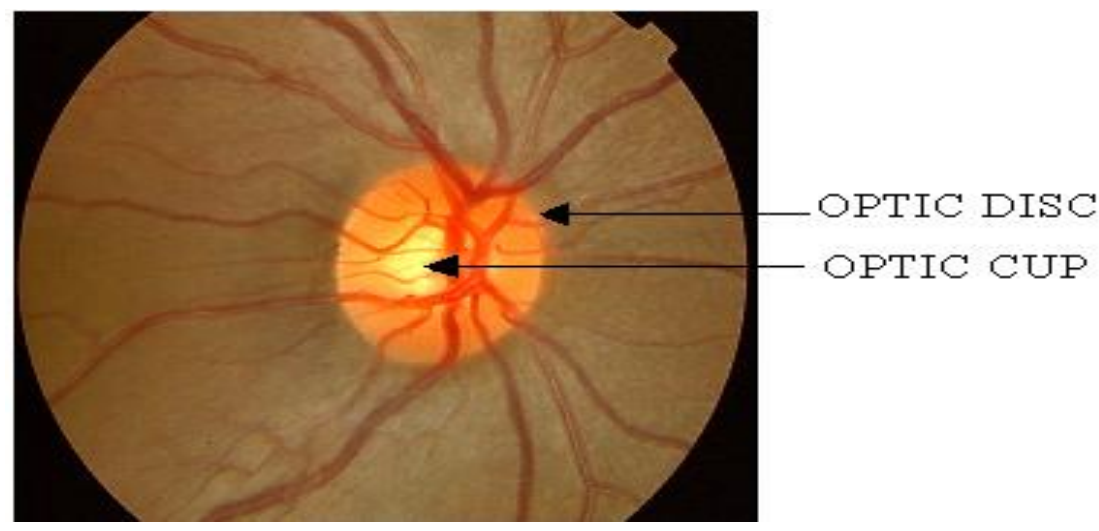

Fig. 1 Fundus Image

In this study, OD is extracted by KMC with HCA [9], and OC is extracted using FCM [10]. The following section gives the methods used for OD and OC segmentation.

\section{METHODS AND MATERIALS}

\section{A. Image Pre-Processing}

In this step, retinal fundus images are preprocessed using anisotropic diffusion [11] filter. The selection of this filter is due to that it does not require the noise pattern, and also it provides contrast. The diffusion equation of this filter is given in Eqn. 1.

$$
I_{t}=\operatorname{div}(c(* x, y, t) \nabla I)=c(x, y, t) \nabla I+\nabla c . \nabla I
$$

Where, $\nabla$ and $\mathrm{c}$ is a gradient and conduction coefficient function respectively. div and $t$ denote the divergence operator and iterations respectively. Figure 2 shows the outputs of preprocessing steps.

\section{B. OD Segmentation}

After preprocessing, it is very important to segment OD region at first and then OC segmentation in order to compute the CDR. Before segmentation of OD, the Region Of Interest (ROI) which contains only OD region is cropped.

\section{B.1 ROI for OD segmentation}

The cropping of ROI region is done automatically based on the intensity of green pixel value in the retinal fundus image. Green pixels provide better contrast than red and blue pixel intensity [12]. The identified maximum intensity points form a cluster of bright region. An ROI of $360 \times 360$ pixels is cropped in the original image around the centre of this cluster. Figure 3 shows the retinal fundus image and its ROI. 


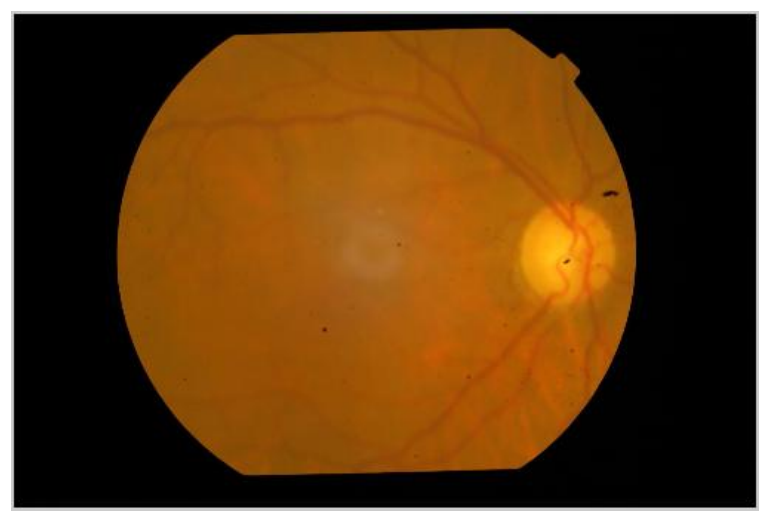

(a) Retinal fundus image

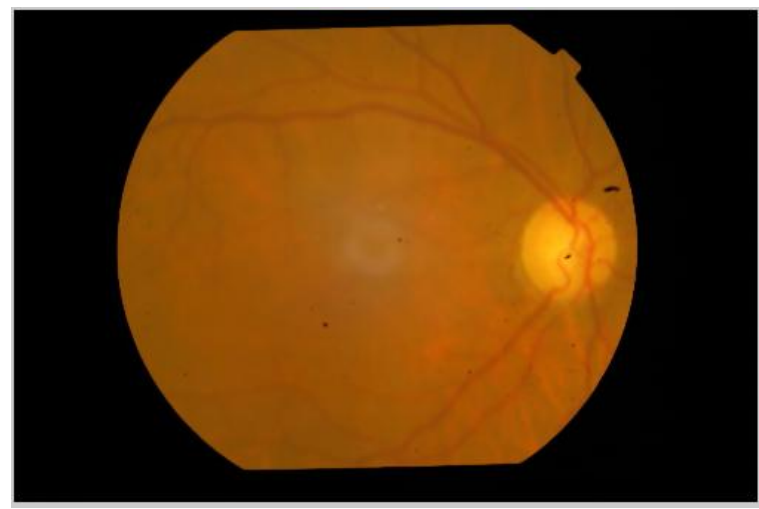

(b) Denoising by anisotropic diffusion

Fig. 2 Preprocessing steps

\section{B.2 Clustering for OD Segmentation}

The system uses KMC for OD segmentation. The accuracy of KMC depends on the number of $k$ seeds. Hence, it is necessary to obtain the value of $k$. HCA is used for this purpose.

\section{B.2.1 HCA to find number of $k$ seeds}

HCA is applied on the ROI of given fundus image. It is operated on CIE $1^{*} a^{*} b^{*}$ image [13]. So that the conversion is taken place using RGB to Lab colour conversion in [14]. Based on the 3D colour histogram, the number of $\mathrm{k}$ is identified. Figure 4 shows the colour histogram of three channels in the Lab colour space separately.

After identifying the number of peaks, KMC is applied on the ROI region. It clusters the data based on ' $\mathrm{k}$ ' value. The OD cluster is identified based on the clustered index of a group of centre pixels. Figure 5 shows the OD segmentation by KMC. 


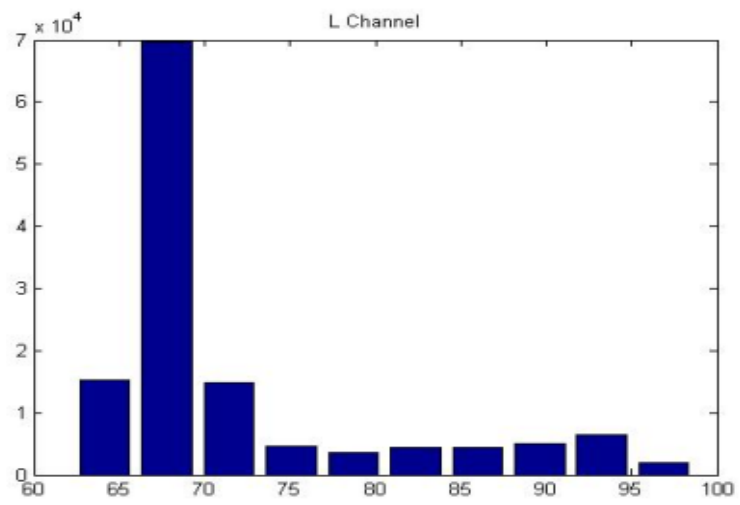

(a) channel ' $L$ '

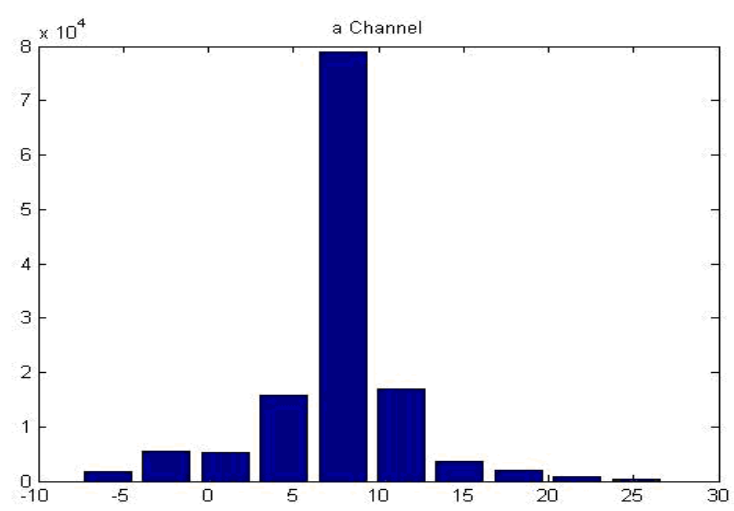

(b) channel 'a'

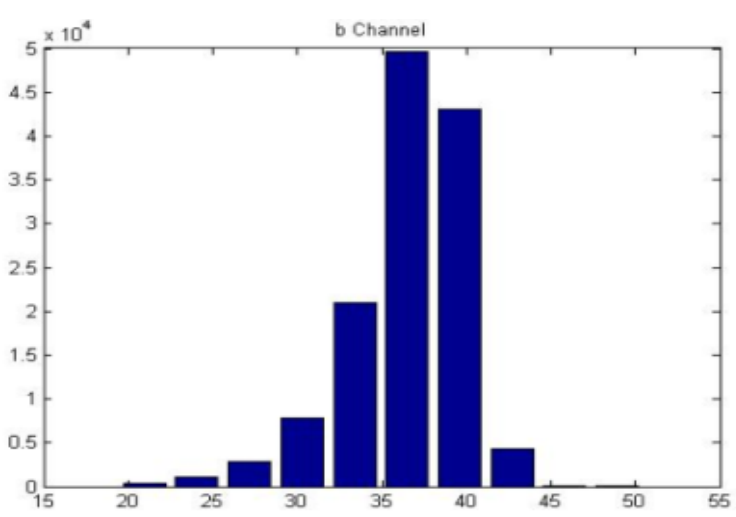

(b) chennal 'b'

Fig. 4 3D Colour Histogram 


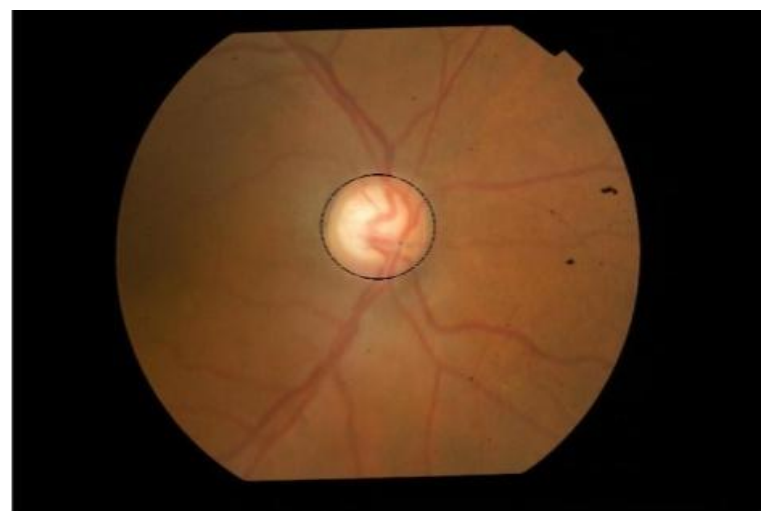

Fig. 5 Segmented Optic Disc

\section{OC Segmentation}

The extraction of $\mathrm{OC}$ is very difficult in comparison with the segmentation of $\mathrm{OD}$ region due to the availability of blood vessels (BVs). OC segmentation consists of two steps; removal of BVs and FCM segmentation.

\section{C.1 Removal of BVs}

Figure 6 shows the steps followed to remove BVs to segment OC efficiently. The input to this step is the segmented OD region. In [16-18], reverse biorthogonal wavelet filter is used to remove the BVs. As this wavelet filter response is same as the structure of BVs, this system also uses this filter for the removal of BVs. Thus, the segmented OD region is only decomposed using one-level decomposition. Using only the low-frequency components for reconstruction, BVs are removed due to that the detail of BV information's available in the highfrequency bands.

\section{C.2 OC segmentation by FCM}

In this system, $\mathrm{FCM}$ is employed for $\mathrm{OC}$ segmentation. $\mathrm{FCM}$ is a supervised clustering approach mainly used for clustering data. The input to FCM is the reconstructed image by IDWT in which all BVs are removed. More information on FCM can be found in [19]. As there is no BVs, the number of clusters is set to 3 (background, OD and OC regions). 


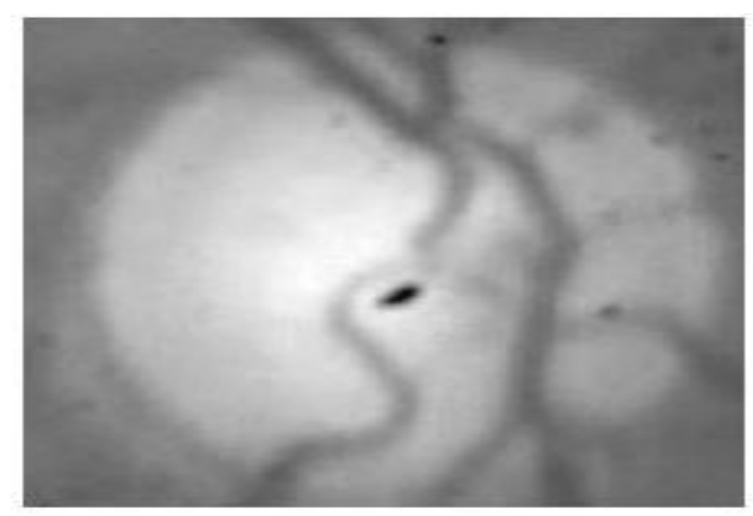

(a) Segmented OC region

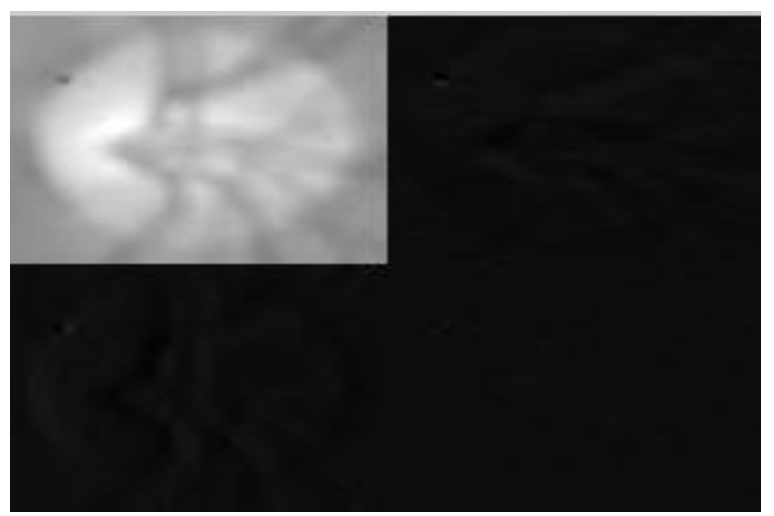

(b) DWT@level 1

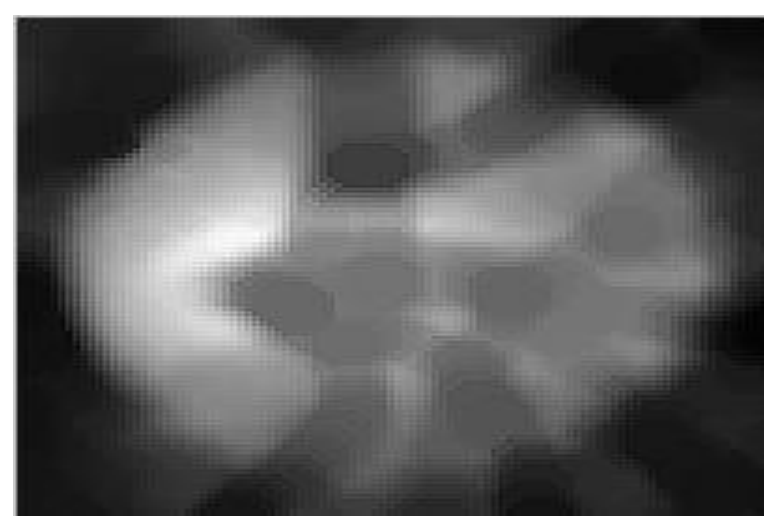

(b) $B V$ removed image

Fig. 6 Steps to remove BVs 


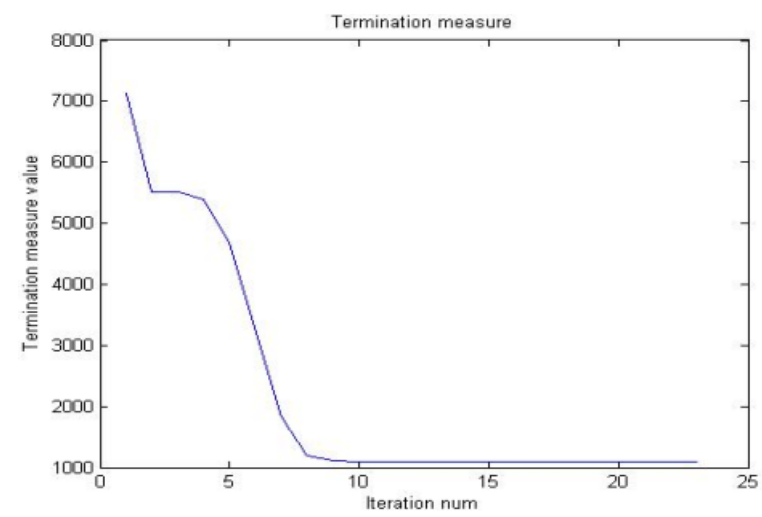

Fig. 7 Iteration vs. termination parameter

The output of FCM gives the OC region which is shown in Figure 8.

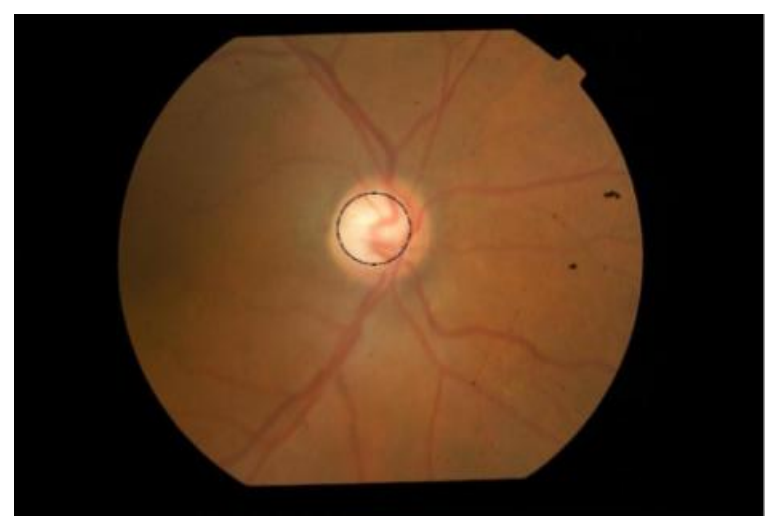

Fig. 8 Optic cup Segmentation

\section{C.3 Computation of OC and OD Diameter}

As the segmented regions are irregular in shape, the computation of OC and OD diameter is achieved by mathematical morphological operations. Figure 9 shows the OD region fully enclosed by a rectangle using connected component analysis.

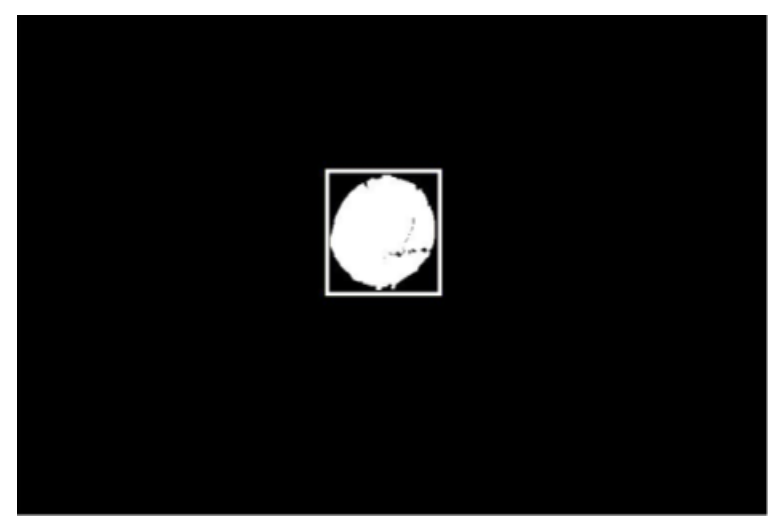

Fig. 9 Rectangle encloses the OD region 
To obtain the CDR, the area of an ellipse that fits inside the rectangle is computed. The area is computed using Eqn. 8.

$$
\text { Area }=\Pi a b
$$

where $a$ and $b$ are the major axis and minor axis length. The above Eqn. 8 is applied on both OD and OC segmented regions so that CDR is easily computed.

\section{RESULTS AND DISCUSSION}

A total of 45 images (20 normal retinal fundus images and 25 abnormal retinal fundus images) obtained from Arvind Eye Hospital, Pondicherry, India is used to test the system. A sample normal retinal fundus image and a sample abnormal fundus image with segmented OD and OC regions are shown in Figure 10 (a) and (b) respectively.

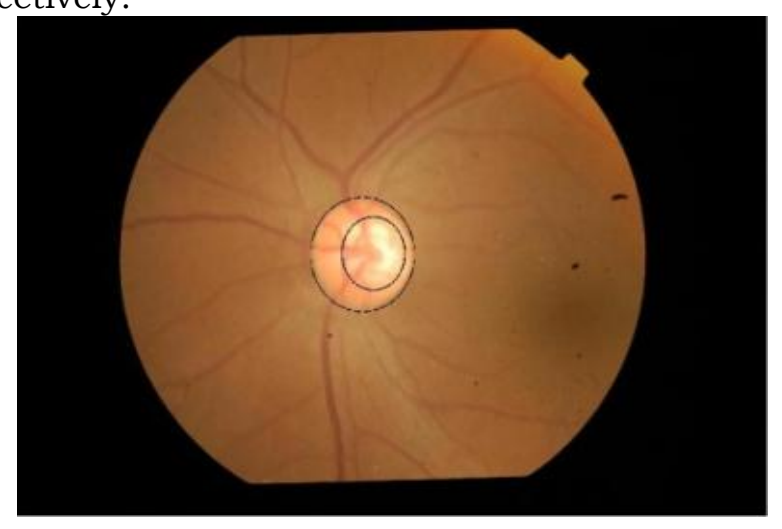

(a) Normal

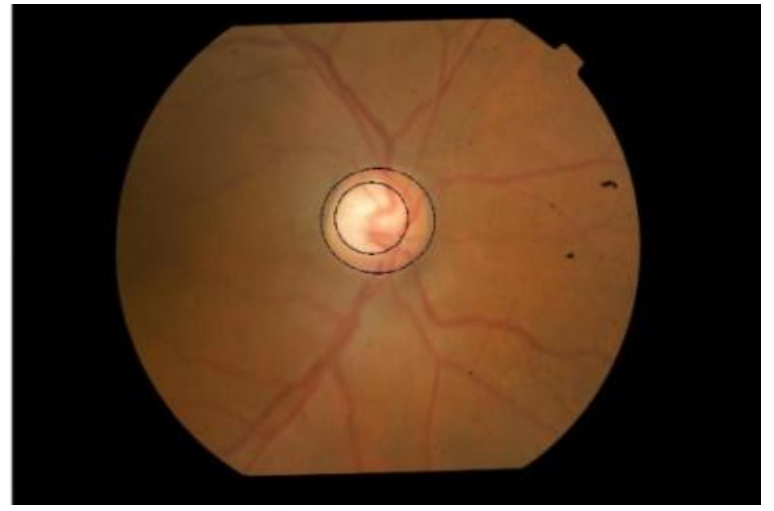

(b) Abnormal

Fig. 10 Fundus image

From the computed area of segmented OC and OD region, CDR is computed for all 45 retinal fundus images and tabulated in Table 1. 

TABLE 1 Computed and gold standard CDR for normal and abnormal
fundus images

\begin{tabular}{|c|c|c|}
\hline $\begin{array}{l}\text { Normal } \\
\text { Fundus } \\
\text { Image }\end{array}$ & $\begin{array}{c}\text { Gold } \\
\text { standard } \\
\text { CDR }\end{array}$ & $\begin{array}{c}\text { Computed } \\
\text { CDR }\end{array}$ \\
\hline 1 & 0.3 & 0.3380 \\
\hline 2 & 0.35 & 0.3437 \\
\hline 3 & 0.3 & 0.2371 \\
\hline 4 & 0.2 & 0.2120 \\
\hline 5 & 0.2 & 0.2391 \\
\hline 6 & 0.35 & 0.3150 \\
\hline 7 & 0.3 & 0.2294 \\
\hline 8 & 0.2 & 0.2451 \\
\hline 9 & 0.3 & 0.3394 \\
\hline 10 & 0.3 & 0.2846 \\
\hline 11 & 0.35 & 0.3346 \\
\hline 12 & 0.3 & 0.3092 \\
\hline 13 & 0.35 & 0.3893 \\
\hline 14 & 0.35 & 0.3886 \\
\hline 15 & 0.3 & 0.3381 \\
\hline 16 & 0.2 & 0.2289 \\
\hline 17 & 0.35 & 0.3907 \\
\hline 18 & 0.2 & 0.2466 \\
\hline 19 & 0.25 & 0.2049 \\
\hline 20 & 0.25 & 0.2044 \\
\hline & & \\
\hline & & \\
\hline & & \\
\hline & & \\
\hline & & \\
\hline
\end{tabular}

\begin{tabular}{|c|c|c|}
\hline $\begin{array}{c}\text { Abnormal } \\
\text { Fundus Image }\end{array}$ & $\begin{array}{c}\text { Gold } \\
\text { standard } \\
\text { CDR }\end{array}$ & $\begin{array}{c}\text { Computed } \\
\text { CDR }\end{array}$ \\
\hline 1 & 0.55 & 0.4952 \\
\hline 2 & 0.45 & 0.4809 \\
\hline 3 & 0.55 & 0.5942 \\
\hline 4 & 0.5 & 0.4742 \\
\hline 5 & 0.5 & 0.4230 \\
\hline 6 & 0.4 & 0.4048 \\
\hline 7 & 0.65 & 0.5276 \\
\hline 8 & 0.4 & 0.4279 \\
\hline 9 & 0.4 & 0.4212 \\
\hline 10 & 0.45 & 0.4279 \\
\hline 11 & 0.4 & 0.4010 \\
\hline 12 & 0.5 & 0.5710 \\
\hline 13 & 0.55 & 0.4553 \\
\hline 14 & 0.45 & 0.4670 \\
\hline 15 & 0.4 & 0.4025 \\
\hline 16 & 0.4 & 0.4063 \\
\hline 17 & 0.5 & 0.4223 \\
\hline 18 & 0.5 & 0.4687 \\
\hline 19 & 0.55 & 0.4625 \\
\hline 20 & 0.6 & 0.4842 \\
\hline 21 & 0.4 & 0.4302 \\
\hline 22 & 0.7 & 0.6401 \\
\hline 23 & 0.55 & 0.4318 \\
\hline 24 & 0.5 & 0.4601 \\
\hline 25 & 0.55 & 0.4725 \\
\hline
\end{tabular}

From Table 1, it is noted that CDR can be effectively used to diagnose glaucoma. A threshold of 0.35 is used for the diagnosis. If the threshold is above 0.35 , the fundus image is said to be abnormal and less than 0.35 , it is said to in a normal condition.

\section{A. Analysis by Scatter Plot}

Figure 11 shows the scatter plot between the computed CDR and gold standard CDR. This plot is drawn using Mintab statistical tool. It produces a Pearson correlation coefficient of 0.917 that shows a high correlation between both CDRs and repeated regression analysis which is less than 0.0001. A linear regression equation is obtained from the plot. It is given in Eqn. 10.

Gold Standard CDR $=-0.0217+1.09$ Computed CDR 


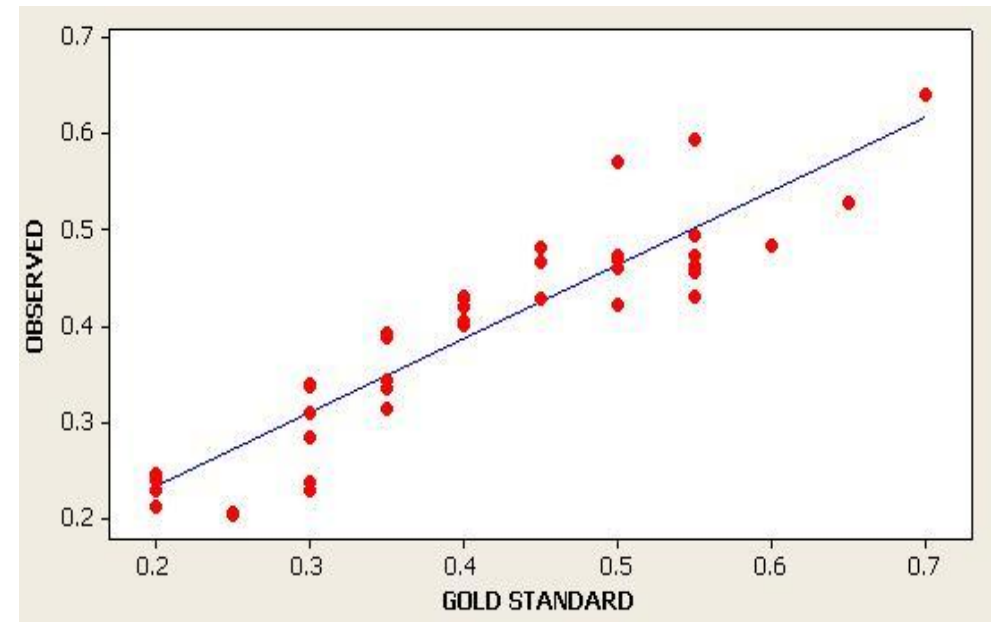

Fig. 11 Scatter plot (gold standard CDR vs. computed CDR)

It is observed from the Figure 11 that there is a small variation in the computed CDR by the system and the ground truth CDR. This is due to the segmentation problem in OC as they are merged with OD sometimes.

\section{CONCLUSION}

In this paper, $\mathrm{CDR}$ is analyzed to indicate the presence of glaucoma using retinal fundus images. KMC and HCA are employed for the segmentation of OD and whereas FCM is used for OC segmentation. The contour of both OD and OC regions are smoothed by the elliptical fitting approach, and then CDR is computed for 45 retinal fundus images. All images are obtained from Arvind Eye Hospital, Pondicherry. It is concluded that if the CDR is greater than 0.35 , the given retinal fundus image is diagnosed with glaucoma and it is less than 0.35; it is a normal eye.

\section{ACKNOWLEDGMENT}

The authors wish to thank Dr. Rengaraj Venkatesh, M.D., Professor of the Aravind Eye Hospital \& Post Graduate Institute of Ophthalmology, Pondicherry, India for providing the expert analysis of the fundus data.

\section{REFERENCES}

[1]. X. Song, K. Song, and Y. Chen, "A computer-based diagnosis system for early glaucoma screening", IEEE 27th Annual International Conference of the Engineering in Medicine and Biology Society, 2006, pp. 6608-6611.

[2]. R. George, and L. Vijaya, "First World Glaucoma day, March 6, 2008 : tackling glaucoma challenges in India", Indian journal of ophthalmology, Vol. 56, No. 2, 2008, pp. 97-98. 
[3]. D. Kavitha, and S. Shenbagadevi, "Automatic detection of optic disc and exudates in retinal images," Proceedings of ICISIP, 2005, pp.501-506.

[4]. Y. Tang, X. Li, A. von Freyberg, and G. Goch, "Automatic segmentation of the papilla in a fundus image based on the CV model and a shape restraint", IEEE $18^{\text {th }}$ International Conference on Pattern Recognition, Vol. 1, 2006, pp. 183-186.

[5]. A.A. Vlachokosta, P.A. Asvestas, G.K. Matsopoulos, N. Uzunoglu, and T.G. Zeyen, "Preliminary study on the association of vessel diameter variation and glaucoma", IEEE 29th Annual International Conference on Engineering in Medicine and Biology Society, 2007, pp. 888-891.

[6]. S. Sekhar, W. Al-Nuaimy, and A.K. Nandi, "Automated localisation of retinal optic disk using Hough transform", IEEE $5^{\text {th }}$ International Symposium on Biomedical Imaging: From Nano to Macro, 2008, pp. 15771580 .

[7]. D.W.K. Wong, J. Liu, J.H. Lim, X. Jia, F. Yin, H. Li, and T.Y. Wong, "Levelset based automatic cup-to-disc ratio determination using retinal fundus images in ARGALI", IEEE 30 th Annual International Conference of Engineering in Medicine and Biology Society, 2008, pp. 2266-2269.

[8]. J. Liu, D.W.K. Wong, J.H. Lim, X. Jia, F. Yin, H. Li, and T.Y. Wong, "Optic cup and disk extraction from retinal fundus images for determination of cup-to-disc ratio", IEEE $3^{\text {rd }}$ Conference on Industrial Electronics and Applications, 2008, pp. 1828-1832.

[9]. T. Ohashi, Z. Aghbari, and A. Makinouchi, "Fast segmentation of texture image regions based on hill-climbing", IEEE Pacific Rim Conference on Communications, Computers and signal Processing, Vol. 2, 2003, pp. 848851.

[10]. R. Krishnapuram, and J.M. Keller, "A possibilistic approach to clustering", IEEE Transactions on Fuzzy Systems, Vol. 1, No. 2, 1993, pp. 98-110.

[11]. P. Perona, and J. Malik, "Scale-space and edge detection using anisotropic diffusion", IEEE Transactions on pattern analysis and machine intelligence, Vol. 12, No. 7, 1990, pp. 629-639.

[12]. Y. Jiang, A. Bainbridge-Smith, and A. B. Morris, "Blood Vessel Tracking in Retinal Images", Proceedings of Image and Vision Computing New Zealand, 2007, pp. 126-131.

[13]. G. Sharma, M.J. Vrhel, and H.J. Trussell, "Digital color Processing", IEEE Transaction on Image Processing, Vol. 6,No. 7, 1997,pp.901-932.

[14]. T. Walter, and J.C. Klein, "Automatic analysis of color fundus photographs and its application to the diagnosis of diabetic retinopathy", In Handbook of Biomedical Image Analysis, Vol. 2, 2005, pp. 315-368.

[15]. A.K. Jain, "Fundamentals of digital image processing", Prentice-Hall, Inc, 4th edition, 1989.

[16]. T. Ohashi, Z. Aghbari, and A. Makinouch, "Hill-climbing algorithm for effcient color-based image segmentation", In IASTED International Conference on Signal Processing, Pattern Recognition, and Applications, 2003, pp.1-5.

[17]. R. Ghaderi, H. Hassanpour, and M. Shahiri, "Retinal vessel segmentation using the 2-D Morlet wavelet and neural network", IEEE International Conference on Intelligent and Advanced Systems, 2007, pp. 1251-1255.

[18]. K. Noronha, J. Nayak, and S.N. Bhat, "Enhancement of retinal fundus image to highlight the features for detection of abnormal eyes", IEEE Region 10 Conference on TENCON, 2006, pp. 1-4.

[19]. N.R. Pal, K. Pal, and J.C. Bezdek, "A mixed c-means clustering model", Proceedings of the $6^{\text {th }}$ IEEE International Conference on Fuzzy Systems, Vol. 1, 1997, pp. 11-21. 